\title{
Genetic analysis of dorsoventral pattern formation in the zebrafish: requirement of a BMP-like ventralizing activity and its dorsal repressor
}

\author{
Matthias Hammerschmidt, ${ }^{1}$ George N. Serbedzija, and Andrew P. McMahon \\ Department of Molecular and Cellular Biology, Harvard University, Cambridge, Massachusetts 02138 USA
}

\begin{abstract}
According to a model based on embryological studies in amphibia, dorsoventral patterning is regulated by the antagonizing function of ventralizing bone morphogenetic proteins (BMPs) and dorsalizing signals generated by Spemann's organizer. Large-scale mutant screens in the zebrafish, Danio rerio, have led to the isolation of two classes of recessive lethal mutations affecting early dorsoventral pattern formation. dino mutant embryos are ventralized, whereas swirl mutants are dorsalized. We show that at early gastrula stages, dino and swirl mutants display an expanded or reduced Bmp4 expression, respectively. The dino and swirl mutant phenotypes both can be phenocopied and rescued by the modulation of BMP signaling in wild-type and

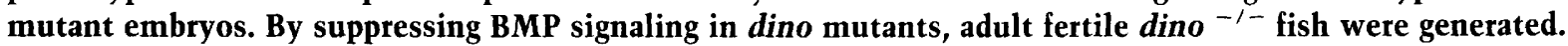
These findings, together with results from the analysis of dino-swirl double mutants, indicate that dino fulfills its dorsalizing activity via a suppression of swirl-dependent, BMP-like ventralizing activities. Finally, cell transplantation experiments show that dino is required on the dorsal side of early gastrula embryos and acts in a non-cell-autonomous fashion. Together, these results provide genetic evidence in support of a mechanism of early dorsoventral patterning that is conserved among vertebrate and invertebrate embryos.
\end{abstract}

[Key Words: Dorsoventral pattern formation; dino; swirl; BMP4; noggin; Spemann's organizer; zebrafish; Danio rerio]

Received June 10, 1996; revised version accepted July 22, 1996.

Our understanding of how the axes of the vertebrate embryo are patterned is dominated by studies of amphibia. Patterning of the anterior-posterior and dorsal-ventral axes of the amphibian embryo is thought to depend on discretely localized signals (for review, see Sive 1993). Maternal signals emanating from vegetal cells induce ventral mesoderm in most of the marginal zone and the dorsal mesoderm of Spemann's organizer in a rather small dorsal region at the site where the formation of the blastopore is initiated. Later, zygotic signals generated by the ventral and dorsal mesoderm themselves regulate the refinement of dorsoventral pattern within the mesoderm and the induction of neuroectoderm in the dorsal animal zone of the early gastrula embryo.

Recent evidence, principally based on studies in Xenopus laevis, supports a simple model of opposing dorsal and ventral activities. Two members of the family of bone morphogenetic proteins (BMPs), members of the TGF $\beta$ superfamily, BMP2 and BMP4, are expressed strongly on the ventral side of early gastrula and have strong ventralizing properties. Overexpression of either

${ }^{1}$ Corresponding author. Present address: Max-Planck-Institut für Immunbiologie, Stuebeweg 51, 79108 Freiburg, Germany. promotes the formation of epidermis and ventral mesoderm (blood islands, mesonephros, mesenchyme) at the expense of neurectoderm and dorsal mesoderm (somites and notochord) (Dale et al. 1992; Jones et al. 1992; Fainsod et al. 1994; Schmidt et al. 1995b; Wilson and Hemmati-Brivanlou 1995; Jones et al. 1996), whereas inhibition of BMP signaling by the expression of a dominant negative receptor (Graff et al. 1994; Maeno et al. 1994; Suzuki et al. 1994; Schmidt et al. 1995b; Sasai et al. 1995), the expression of cleavage resistant mutant forms of BMPs (Hawley et al. 1995), or the injection of Bmpantisense RNA (Sasai et al. 1995; Steinbeisser et al. 1995) leads to a dorsalized phenotype. The antisense experiments, which discriminate between BMP2 and BMP4, suggest that it is BMP4 that is actually required for ventral development (Sasai et al. 1995; Steinbeisser et al. 1995).

Dorsal development depends on signals emanating from the Spemann's organizer region on the dorsal side of the gastrulating embryo. Three secreted peptides, Noggin, Chordin, and Follistatin, have been identified that are synthesized in the organizer and that promote the formation of dorsal mesoderm and neuroectoderm (Smith and Harland, 1992; Sasai et al. 1994; Hemmati- 
Brivanlou et al. 1994). Thus, these factors have opposing activities to, and are counteracted by BMPs (Sasai et al. 1995; Jones et al. 1996). Follistatin binds activins, which are also members of the TGF $\beta$ superfamily, preventing activin signaling (Nakamaru et al. 1989). Recent evidence indicates that Chordin and Noggin also bind BMPs with high affinity, thereby preventing the interaction of BMPs with their receptors (Piccolo et al. 1996; Zimmerman and Harland 1996). Thus, the induction of specific dorsoventral positional identities may depend upon the modulation of signaling by BMPs, through the direct blocking action of dorsally localized antagonists.

Interestingly, Chordin shares structural and functional homology with Drosophila Short gastrulation (Sog), which acts as an antagonist of Decapentaplegic (Dpp), the Drosophila BMP2/4 homologue, during patterning of the dorsoventral axis of the Drosophila embryo (Ferguson and Anderson 1992b; Wharton et al. 1993; François et al. 1994). The opposing distributions and activities of Chordin and BMP4 in Xenopus and Sog and Dpp in Drosophila suggest a common mechanism for dorsoventral patterning in bilateral metazoa (François and Bier 1995; Jones and Smith 1995; DeRobertis and Sasai 1996).

While the relevance of the fly genes for normal dorsoventral pattern formation in vivo has been demonstrated in loss-of-function mutants, similar studies of the vertebrate genes are ambiguous. Gene targeting experiments in the mouse have revealed the general requirement for BMP4 and a type I BMP receptor during early mouse gastrulation and mesoderm formation (Mishina et al. 1995; Winnier et al. 1995). Homozygous mutant mouse embryos display variable phenotypes. Severely affected mutants die during early gastrulation stages and form no or only little mesoderm, making it difficult to address the proposed role of BMP4 signaling during the refinement of mesodermal patterning and neural induction. Those embryos with the least severe phenotype die at early somite stages and have truncations of the posterior axis that might be interpreted as ventral deficiencies.

Recently, screens for randomly introduced mutations in the zebrafish (Haffter et al. 1996) have led to the identification of two distinct classes of mutants that display an altered dorsoventral pattern, either a general ventralization or a general dorsalization. In mutants of both classes, the dorsalmost mesoderm of the shield, which is the fish equivalent of Spemann's organizer in amphibia (Ho 1992; Shih and Fraser 1996), is formed normally. However, the mutants display severe alterations in the patterning of regions outside the organizer. Mutants in dino are ventralized and display an enlargement of ventrolateral mesodermal fates and a reduction of neuroectodermal and dorsolateral mesodermal fates (Hammerschmidt et al. 1996b). In contrast, swirl mutants are dorsalized and show the opposite phenotype, an enlargement of the neuroectoderm and dorsolateral mesodermal fates, and a reduction of ventrolateral mesodermal fates (Mullins et al. 1996). The phenotypes of the mutants suggest that dino and swirl are required for the zygotic control of dorsoventral pattern formation after the initial establishment of a dorsoventral polarity, which appears to be under maternal control. The phenotypes are consistent with the model of counteracting zygotic ventralizing and dorsalizing activities drawn from the aforementioned studies in amphibia.

We provide several lines of evidence indicating that the swirl and dino mutant phenotypes result from a modulation of normal BMP signaling. The dorsalizing gene encoded by dino appears to be required on the dorsal side of the pregastrula embryo to suppress a ventralizing swirl-dependent BMP signal. Thus, these genetic studies indicate that zygotic regulation of the vertebrate body plan does have a similar underlying mechanism to that of the Drosophila embryo.

\section{Results}

\section{Morphology of dino and swirl mutant embryos}

As previously described (Hammerschmidt et al. 1996b), dino mutants appear ventralized, displaying a general shift in the dorsoventral organization of the mesoderm. Dorsolateral cell fates are suppressed, the posterior notochord is lost, and the anterior somites are reduced (Fig. $|B, E|$, while the blood and pronephros, which derive from more ventral mesoderm, are expanded. In contrast, swirl mutants appear dorsalized. The notochord is broader, the somites extend laterally, and blood and pronephric development is absent (Mullins et al. 1996; Fig. 1C,F F. In addition, dino mutants display a general enlargement of posterior structures at the expense of anterior structures, and an overall reduction of the neuroectoderm, whereas swirl mutants have the opposite phenotype (Figs. 1A-F, 2D,E, 3F,G). Neither mutant is viable: swirl mutants die within $24 \mathrm{hr}$ after fertilization, and dino mutants at later stages.

\section{Expression of Bmp4 in dino and swirl mutants}

To study whether alteration of BMP4 might underlie the dino and swirl phenotypes, we examined the $B m p 4$ expression in these mutants. At late blastula stages, $B m p 4$ is uniformly expressed in both wild-type and mutant embryos (not shown). Starting shortly before gastrulation, $B m p 4$ expression is progressively lost in dorsolateral regions of wild-type embryos. Expression is maintained in ventrolateral regions and in the anterior dorsal endomesoderm (Fig. 1G,J). In contrast, in dino mutant embryos $B m p 4$ expression remains strong in all regions (Fig. $1 \mathrm{H}, \mathrm{K})$, while in swirl mutants, Bmp4 expression is lost everywhere except the anterior endomesoderm (Fig. $1 I, \mathrm{~L})$. This suggests that dino is required for the repression of $B m p 4$ expression in dorsolateral regions, while swirl is required for the maintenance of ventrolateral Bmp4 expression. Further, the observed alterations in the expression pattern of $B m p 4$ appear to be consistent with, and might account for, the altered dorsoventral pattern seen in dino and swirl mutant embryos. To test this notion, we studied whether the dino and swirl mutant phenotypes can be phenocopied and rescued by al- 


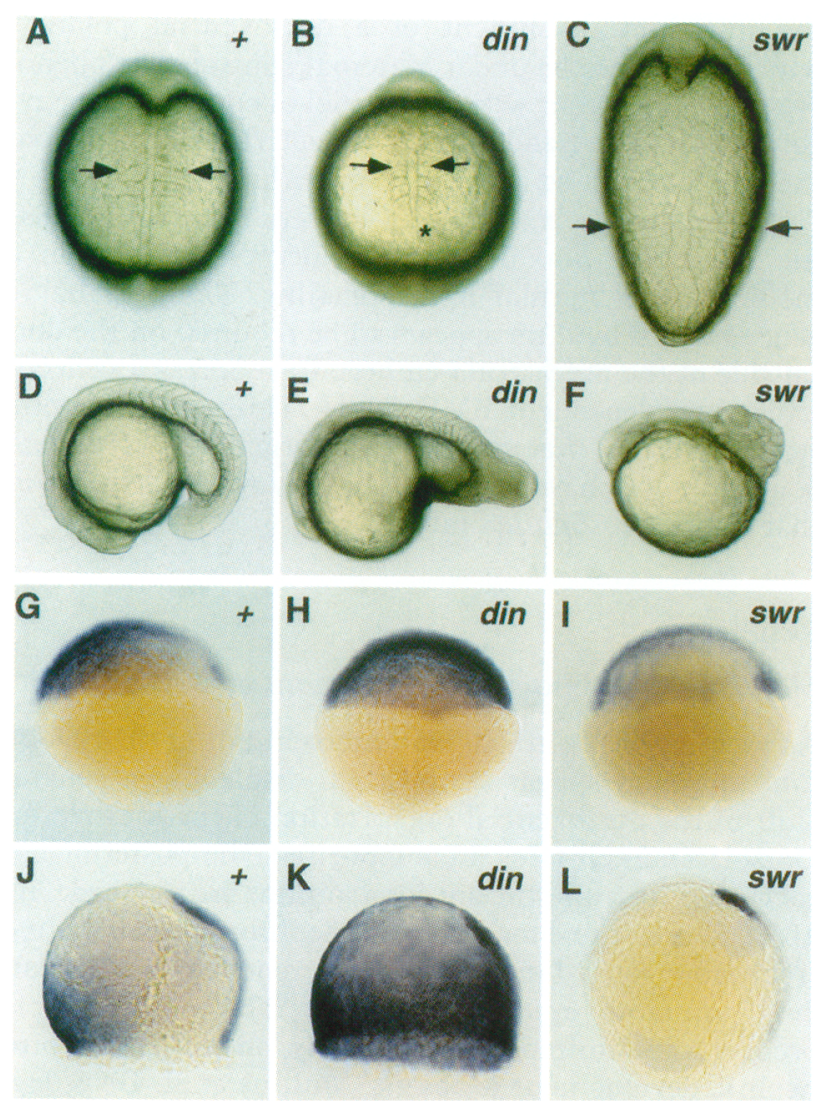

Figure 1. dino and swirl mutant zebrafish embryos: morphology and Bmp4 expression. $(A, D, G, I)$ Wild-type; $(B, E, H, K)$ dino mutant; $(C, F, I, L)$ swirl mutant. $(A-C)$ Three-somite stage, dorsal view. The arrows point to the first somites as a reference point to assess the anteroposterior alterations. In $B$, the loss of the notochord is indicated by an asterisk. $(D-F) 15$-Somite stage, lateral view; $(G-L) B m p 4$ expression; $(G-I)$ shield stage; $(I-L)$ $80 \%$ epiboly, lateral view, dorsal right. (+ | Wild-type.

tering BMP signaling in wild-type or mutant embryos, respectively.

\section{Phenocopy of dino mutant phenotype by Bmp4 overexpression}

To test the hypothesis that the ventralization of dino mutants is caused by the expanded $B m p 4$ expression, we studied whether overexpression of $B m p 4$ in wild-type embryos leads to a dino-like phenotype. While embryos injected with control plasmid (pCSKA-lacZ; Sasai et al. 1995) showed no sign of ventralization (0/63, not shown), embryos injected with a similar construct expressing Bmp4 (pCSKA-BMP4) displayed most phenotypic traits of dino mutant embryos $(250 / 423,59 \%)$. As in dino mutants, the derivatives of Spemann's organizer appear normal in pCSKA-BMP4-injected wild-type embryos, as revealed by the expression of gsc (Stachel et al. 1993; Schulte-Merker et al. 1994; Thisse et al. 1994) in presumptive prechordal plate cells (Fig. 2G-I) and the ex- pression of the notochord marker gene ntl (SchulteMerker et al. 1992) in the anterior part of the axis (Fig. 2J-L). However, dorsal fates outside the organizer are reduced, as shown by the reduced size of the expression domain of the neuroectodermal marker gene $f k d 3$ (Fig. 2D-F; cf. Hammerschmidt et al. 1996b/ and the lack of $n t l$ expression in the posterior part of the axis (Fig. 2J-L). In contrast, ventrolateral fates are expanded, as revealed by the broader expression patterns of the ventrolateral marker gene eve1 at gastrula stages (Fig. 2G-I; Joly et al. 1993) and the hematopoietic marker gene gata1 at segmentation stages (Fig. 2J-L; Detrich et al. 1995). Therefore, it is likely that the altered dorsoventral organiza-

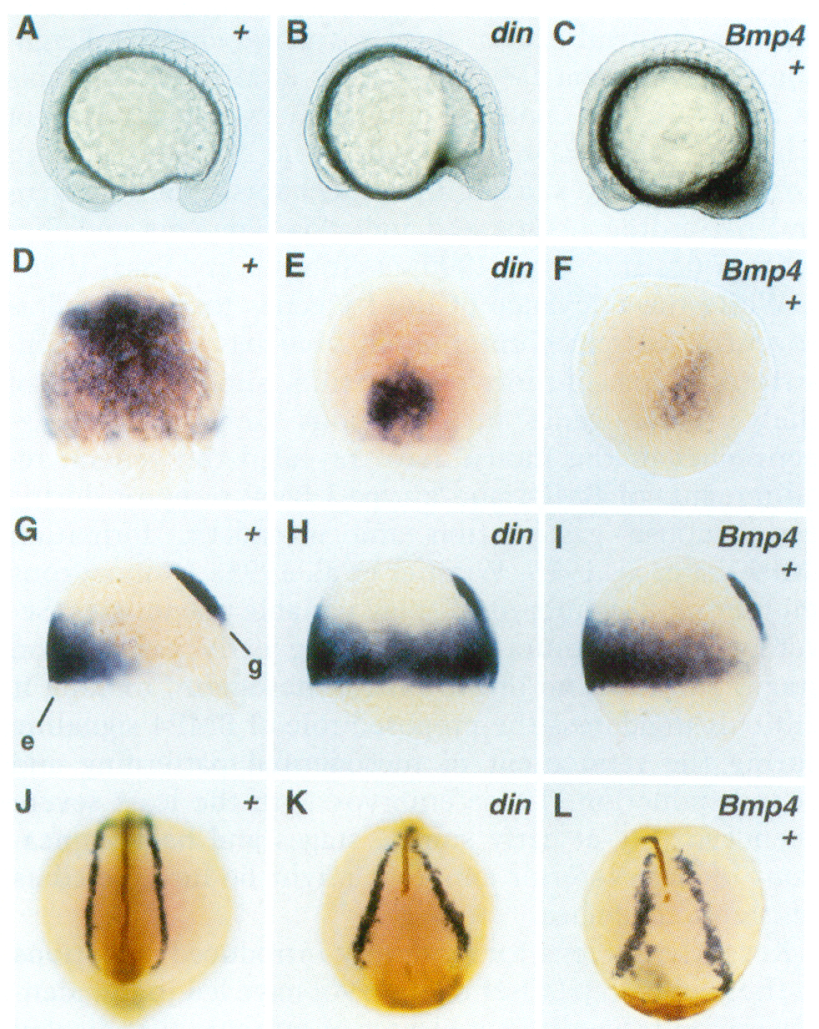

Figure 2. The dino mutant phenotype is copied in wild-type embryos by $B m p 4$ overexpression. $(A, D, G, J)$ Wild-type; $(B, E, H, K)$ dino mutant; $(C, F, I, L)$ wild-type injected with pCSKA-BMP4. $(A-C) 15$-somite stage; $(D-F)$ expression of $f k d 3$ in presumptive neuroectoderm, $80 \%$ epiboly, dorsal view; $(G-I)$ expression of $g s c(\mathrm{~g})$ and eve1 (e), 70\% epiboly, lateral view, dorsal right; $(I-L)$ expression of gata1 (blue) and $n t 1$ (brown), 10 -somite stage, dorsal view. Note the expansion of the gata1 expression domains in the two lateral stripes. The more posterior gata1 expression is delayed in both the mutant and the injected wild-type embryo. At the 15-somite stage, however, this posterior expression domain will also be much broader than in uninjected wild-type embryos (not shown, but see Hammerschmidt et al. 1996b). Also note the increased size of the tissues interposed between the two gata1 stripes posteriorly and the closer apposition anteriorly, which reflects the general enlargement of the posterior region at the expense of the anterior region of the embryo pointed out in Fig. 1. 
A
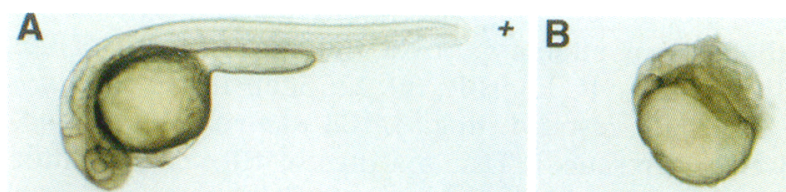

C
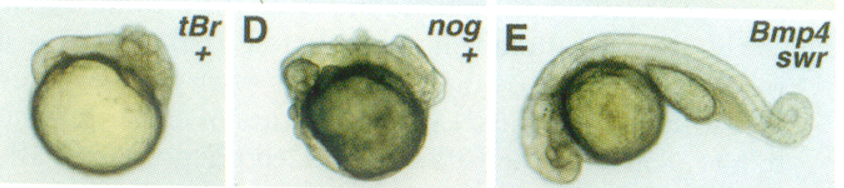

F

$+\mathrm{G}$
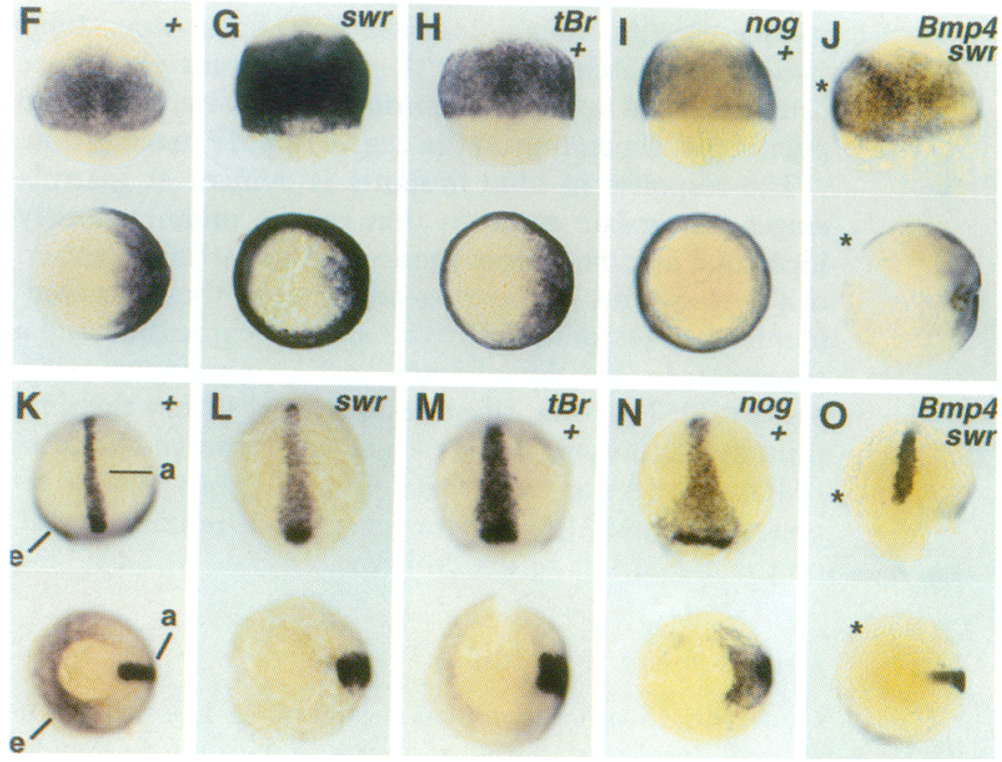

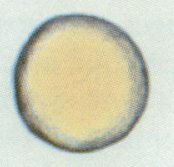

*

swr
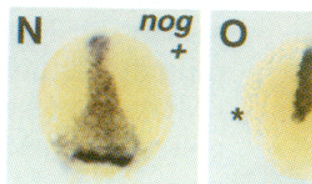

*

Figure 3. The swirl mutant phenotype is copied in wild-type embryos by the injection of synthetic RNA encoding a dominant negative truncated form of the Xenopus BMP receptor $(\mathrm{tBr})$ or Noggin (nog), and rescued in swirl mutants by expression of $B m p 4$. $(A-E)$ 1.5-day-old embryos; the rescued embryo in $E$ displays a "piggy-tail"-like phenotype, indicative of a weak dorsalization (Mullins et al. 1996). $(F-I) f k d 3$ expression, $70 \%$ epiboly; $(K-N)$ expression of $a x 1(\mathrm{a})$ and eve $1(\mathrm{e}), 80 \%$ epiboly. $(A, F, K)$ Uninjected wildtype; $(B, G, L)$ uninjected swirl mutant; $(C, H, M) \mathrm{tBr}-$ injected wild-type; $(D, I, N)$ nog-injected wild-type; $(E, J, O)$ Bmp4-injected swirl mutant. $F-O$ show a dorsal view on the top and an animal view $(F-J)$ or vegetal view $(K-O)$ with dorsal right on the bottom. In $I$ and $O$, sites with some remaining swirl-specific expression of $f k d 3$ and $e v e 1$ are marked *.

tion of dino mutant embryos results from the observed dorsal expansion of $B m p 4$ expression. In the wild-type, dino presumably acts as an antagonist of $B m p 4$ expression in the dorsolateral region of the gastrulating embryo.

\section{Phenocopy of swirl mutant phenotype by BMP inactivation}

To determine whether the loss of $B m p 4$ expression in swirl embryos leads to a dorsalized phenotype, we inactivated BMP4 in wild-type embryos (and potentially other related BMPs that might be expressed) by two different means: the injection of synthetic RNA encoding either a truncated, dominant negative form of the Xenopus BMP receptor $(\mathrm{tBr}$; Graff et al. 1994; Maeno et al. 1994; Suzuki et al. 1994; Schmidt et al. 1995b) or Xenopus Noggin, which binds to BMP2 and BMP4 at picomolar concentrations, preventing the binding of the BMPs to their receptor (Zimmerman and Harland 1996). As in the Xenopus embryo, injection of $\mathrm{tBr}$ mRNA $150-100 \mathrm{pg}$ per embryo, 117/119, 98\%) or noggin mRNA (2-5 pg, $98 / 98,100 \%$ ) into wild-type zebrafish embryos caused a dorsalization. Injected embryos look very similar to dorsalized zebrafish mutants (Mullins et al. 1996), with the body axis wound up in a snailshell-like fashion (Fig. 3B-
D). With molecular markers, the dorsalization is already apparent at midgastrula stages. As in swirl mutants (Fig. $4 G, L)$, expression of the neuroectodermal marker gene $f k d 3$ extends into ventralmost regions (Fig. $3 \mathrm{H}, \mathrm{I}$ ), and the expression domain of the notochord marker gene axial (axl) (Strähle et al. 1993) is much broader. Moreover, expression of eve1, a ventrolateral marker, is absent (Fig. $4 \mathrm{M}, \mathrm{N}$ ). This almost perfect phenocopy of the swirl mutant phenotype by suppression of ventral BMP signaling in wild-type embryos suggests that the phenotype of the mutant embryos might be caused by the observed loss of endogenous BMP signaling.

Partial rescue of swirl and dino phenotypes by modulation of BMP signaling

If altered BMP signaling is responsible for the dino and swirl phenotypes, it should be possible to rescue mutant embryos by modifying BMP signaling. To test this prediction we intercrossed swirl heterozygotes and injected their embryos with pCSKA-BMP4 to drive ectopic Bmp4 expression. Significantly less than a quarter of the embryos $(11 / 303,3.6 \%)$ displayed the swirl-specific football-like shape (see Fig. 1C) at the end of gastrulation. Moreover, at later stages, some embryos (17/303) showed only a weakly dorsalized phenotype (Fig. 3E; 


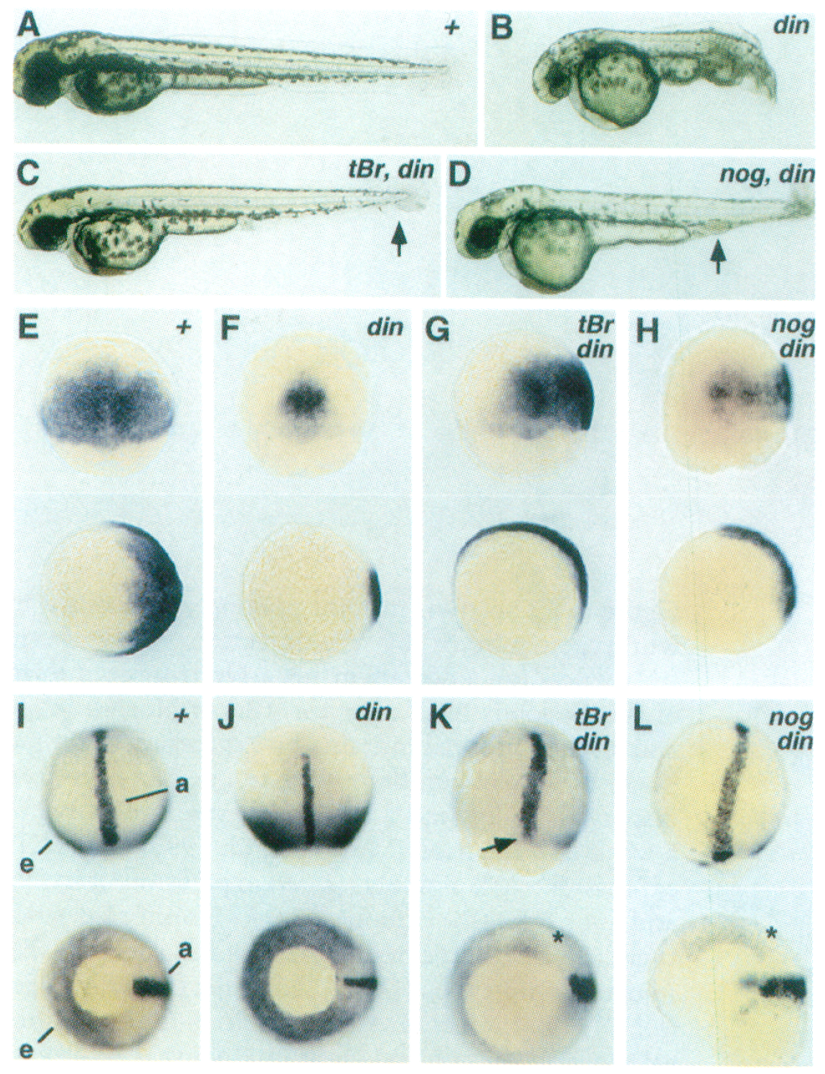

Figure 4. dino mutants are rescued by the injection of synthetic $\mathrm{tBr}$ or nog mRNA. $(A-D)$ Two-day old larvae; $(E-H) f k d 3$ expression, $70 \%$ epiboly; $(I-L)$ expression of $a x l(a)$ and eve1 (e), $80 \%$ epiboly. $\{A, E, I\rangle$ Uninjected wild-type; $\{B, F, I\rangle$ uninjected dino mutant; $\{C, G, K) \mathrm{tBr}$-injected dino mutant; $\{D, H, L)$ noginjected dino mutant. $E-L$ show a dorsal view on the top and an animal view $(E-H)$ or vegetal view $(I-L)$ with dorsal right on the bottom. The arrow in $C$ points to a slight duplication of the ventral tail, the arrow in $D$ to extra cells in the blood islands, characteristics of dino mutants. The embryo in $C$ was raised and shown to be a dino homozygote by segregation analysis. The embryos in $G$ and $H$ show a dino-like $f k d 3$ expression pattern on the left side, and an expanded $f k d 3$ expression on the right side of the embryo. In $K$ and $L$, sites with some remaining dino-specific expression of $a x l$ and $e v e 1$ are marked $\uparrow$ and *, respectively.

Mullins et al. 1996). The remaining, nonidentifiable swirl mutant embryos in these experiments were probably in the fraction that were ventralized or that appeared to be wild-type. In embryos that had maintained a swirl-specific marker gene expression pattern in some regions of the embryo, and that were therefore identifiable as swirl homozygotes, we observed a suppression of $f k d 3$ and axl expression and some restoration of eve1 expression (Fig. 3J,O). Thus, expression of $B m p 4$ can partially rescue the swirl mutant phenotype, although we have so far failed to identify viable swirl homozygotes.

In contrast, we were able to recover adult and fertile dino $^{-1-}$ mutants after the inactivation of BMPs by injection of either $\mathrm{tBr}$ RNA or noggin RNA. At larval stages, the injected mutants exhibited a dramatic rescue of the mutant phenotype over the entire length of the body axis (Fig. 4C,D) (tBR: 39/225 identifiable as dino, 30/39 clearly rescued; nog: 3/103 identifiable as dino, $2 / 3$ clearly rescued). Test matings of $\mathrm{tBr}$-rescued adult mutants confirmed their identity as dino homozygotes as the mutant allele was transmitted to all offspring (6/ 6). Rescue was dose-dependent. As with wild-type embryos, injection of increasing amounts of either RNA led to a typical snailshell-like dorsalized phenotype in all of the injected embryos derived from dino heterozygous intercrosses (tBr: 200pg, 115/122 dorsalized; nog: 20pg, $246 / 246$ dorsalized). Thus, the dino mutant phenotype can be rescued or converted into the opposite, dorsalized phenotype, depending on the degree of BMP inactivation.

The response of dino mutants is evident at gastrula stages. In mosaic embryos that can be unambiguously identified as dino homozygotes by the partial retention of a dino-specific gene expression pattern, there is a complementary restoration of $f k d 3$ and $a x l$ expression and a suppression of dorsolateral eve1 expression (Fig. $4 \mathrm{G}, \mathrm{H}, \mathrm{K}, \mathrm{L})$. Together, these results suggest that the dino mutant phenotype results from the observed lack of BMP4 suppression, and that Dino functions via the repression of BMP4, and/or a related ventralizing signal, on the dorsal side of the embryo.

\section{swirl is epistatic to dino}

Genetic data supporting this model come from the analysis of dino-swirl double mutants. According to Mendelian segregation of two unlinked loci, $3 / 16(18.75 \%)$ of the offspring from a cross of dino $/+$, swirl + double heterozygous parents should be homozygous for dino, $3 / 16$ homozygous for swirl, and $1 / 16(6.25 \%)$ homozygous for both dino and swirl. However, we found that, rather than $18.75 \%$, approximately one-quarter of the offspring displayed the dorsalized phenotype typical for swirl single mutants $(375 / 1508,24.9 \%, 10$ crosses $)$, while dino mutant embryos occurred in the expected proportion (173/930, 18.6\%, 8 crosses). No new doublemutant category was observed. The same distribution of phenotypes was found at gastrula stages when embryos were stained with molecular markers $39 / 140$ were dorsalized), indicating that the dino-swirl double mutant has the same phenotype as the swirl single mutant, and that swirl is epistatic to dino. Clearly, if Dino acts as a repressor of BMPs, there would be no requirement for dino activity in a swirl mutant background in which the ventralizing BMP activity is missing from early gastrula stages (Fig. 1I,L).

Interestingly, the partial loss of the swirl-dependent ventralizing activity seems to compensate for the loss of dino. While embryos of the swirl category were uniformly dorsalized, dino mutant embryos displayed significant differences in the strength of their phenotype. Only $8.2 \%$ (77/930) embryos showed the strong dino phenotype normally found in a cross of two dino single heterozygotes (Fig. 5B,F,H,J), while $11.4 \%$ (106/930) showed a significantly weaker phenotype. Morphologi- 


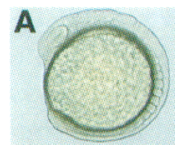

E

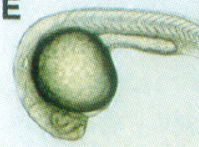

H

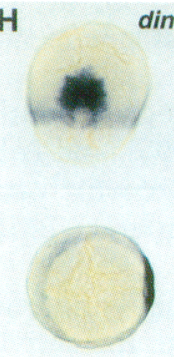

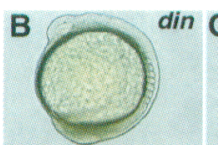

$+F$

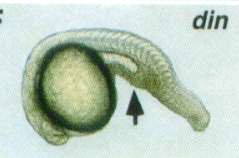

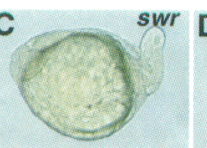

G

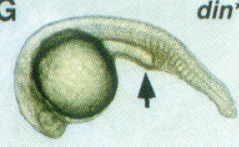

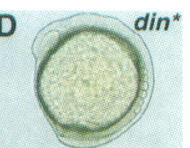

$\operatorname{din}^{*}$

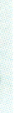
$i n^{*}$
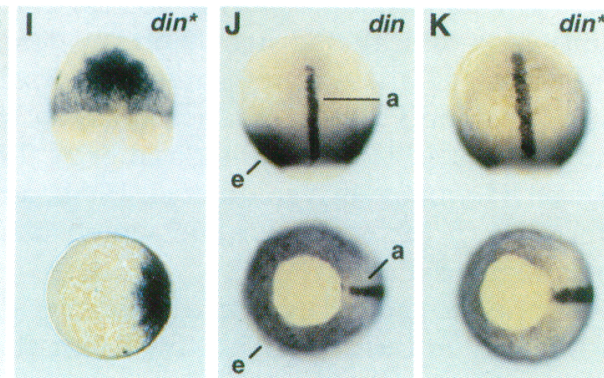

Figure 5. dino-swirl double-mutant analysis. $(A-D)$ eightsomite stage; $(E-G) 24$ hr. $(A, E)$ Wild-type; $(B, F)$ dino mutant; (C) swirl mutant; $(D, G)$ partially rescued dino mutant * (most likely $\operatorname{din}^{-1-}$, swr ${ }^{+/-} \mid$. In comparison to $B$ and $F$, the size of the eyes in $D$ and $G$ is increased, and fewer cells, indicated with arrows, are present in the posterior, ventral part of the axis. $(H, I)$ $f k d 3$ expression, $70 \%$ epiboly; $(J, K)$ expression of $a x l$ (a) and eve 1 (e), $80 \%$ epiboly. $(H, I)$ dino mutant; $(I, K)$ partially rescued dino mutant ${ }^{*} . H-K$ show a dorsal view on the top and an animal view $(H, I)$ or vegetal view $(J, K)$ with dorsal right on the bottom. The expression patterns in $I, K$ are more similar to wild-type (Fig. 4E,I) than those found in regular dino mutants $(H, J)$.

cally, these embryos showed enhanced development of the anterior central nervous system, in particular, the eyes, and a reduction in posterior ventral development (Fig. 5D,G). At gastrulation stages, we observed an expanded domain of $f k d 3$ and $a x l$ expression together with a reduction of dorsolateral eve1 expression (Fig. 5I,K). Although proof would require accurate genotyping, a simple explanation consistent with the aforementioned data is that dino mutants with the weaker phenotype were heterozygous for the mutant swirl allele (expected for $12.5 \%$ of the offspring), while dino mutants with the stronger ventralized phenotype had two wild-type swirl alleles (expected for $6.25 \%$ ). The comparison of the expected and the observed segregation pattern passes the chi-square test $\left(\chi^{2}=6.1, P<0.10\right)$. Thus, the partial loss or inactivation of swirl or BMP4 (by injection of RNA encoding the dominant negative BMP receptor or Noggin) leads to a rescue of the dino mutant phenotype, while the complete loss or inactivation turns it into the opposite, dorsalized phenotype. This suggests that the dino-swirl/BMP4 counteraction is dose-dependent.

Dino is required on the dorsal side and functions in a non-cell-autonomous fashion

To further address the role of Dino as a dorsal repressor of ventralizing activities, we performed cell transplanta- tion experiments. Labeled wild-type cells were transplanted into embryos from a dino heterozygous intercross at both the sphere stage, prior to gastrulation, when the dorsal side cannot be identified in donors and hosts, and at the shield stage, shortly after the onset of gastrulation, when wild-type cells can be taken from and transplanted into defined dorsoventral positions. In all sets of transplantation experiments, the fraction of normal chimeric embryos was close to $75 \%(110 / 144)$, suggesting that a complete rescue of dino mutants did not occur. However, most mutant chimeric embryos with a successful dorsal transplantation of wild-type cells showed a significantly weaker phenotype than nonchimeric mutant siblings, when the cells were transplanted at either the sphere $\left(4 / 5\right.$, Fig. $6 C$ ) or shield stage $\left(d \rightarrow d_{i} 8 / 11\right.$, Fig. $6 \mathrm{E}, \mathrm{F})$. In contrast, transplantation of wild-type cells to the ventral side of host embryos had no effect, regardless of whether they were taken from the dorsal or the ventral side at the shield stage $(\mathrm{d} \rightarrow \mathrm{v}, 0 / 5 ; \mathrm{v} \rightarrow \mathrm{v}, 0 / 4$; not shown) or at the sphere stage $(0 / 9$, Fig. $6 \mathrm{D})$.

The transplantation of dorsal wild-type cells into the

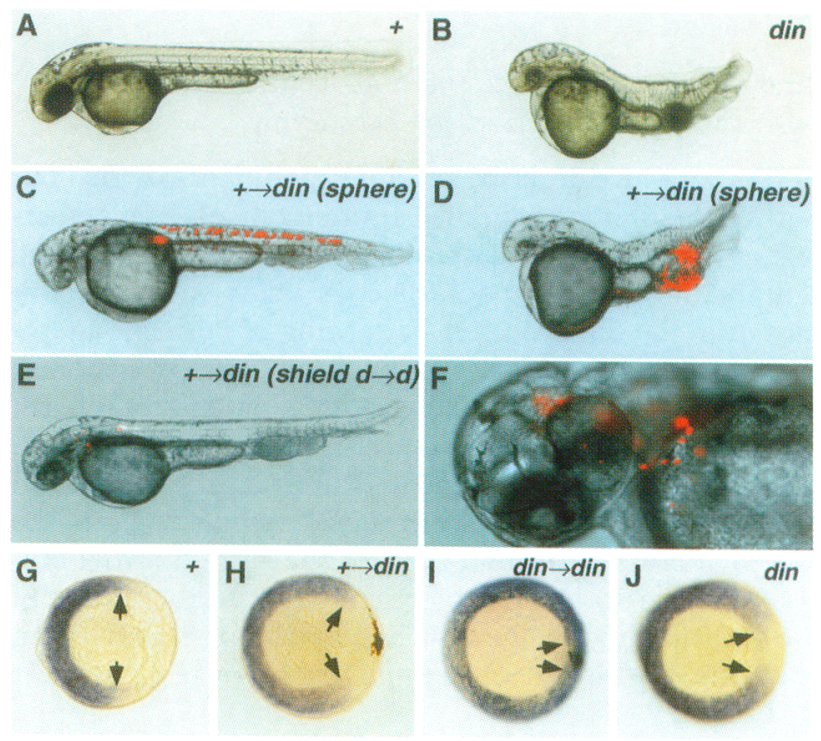

Figure 6. The dino mutant phenotype is rescued by wild-type cells transplanted to the dorsal side of pregastrula or early gastrula mutant embryos. $(A-F)$ Two-day-old larvae. $(A)$ Wild-type; (B) dino; $(C-F)$ wild-type $\rightarrow$ dino chimeras, wild-type cells labeled in red; $(C, D)$ transplantation at sphere stage. (C) Partially rescued, wild-type cells in spinal cord, somites, and posterior notochord; no wild-type cells in hatching glands and anterior notochord, which might explain the nonrescued head phenotype (note small eyes); $(D)$ not rescued, wild-type cells in blood islands and ventral part of posterior somites. $(E, F)$ Transplantation at shield stage, partially rescued, wild-type cells in hatching glands and head mesenchym. In $F$, a magnification of $E$ is shown. $(G-I)$ eve1 expression, $80 \%$ epiboly, vegetal view, dorsal right; the dorsal borders of the eve1 expression domain are marked by arrows. $(G)$ Wild-type; $(H, I)$ chimeras after transplantation at shield stage; transplanted wild-type cells are labeled in brown. $(H)$ Wild-type $(\mathrm{d}) \rightarrow$ dino $(\mathrm{d}) ; \quad(I)$ dino $(\mathrm{d}) \rightarrow$ dino $(\mathrm{d}) ; \quad(J)$ dino, weak phenotype. 
dorsal shield normally led to chimeric embryos with rather few $(50-100)$ wild-type cells restricted to anterior structures of the chimeric larvae (Fig. 6E,F). Nevertheless, the phenotype of these chimeras appeared normalized over the entire length of the axis. The normalizing effect of transplanted wild-type cells was already apparent at gastrula stages. Chimeric mutant embryos, with wild-type cells in the region of the presumptive prechordal plate and the notochord, displayed a significant retraction of the expression of the ventrolateral marker gene eve 1 in regions that are completely devoid of wildtype cells (19/31, Fig. 6H). A weaker, unilateral retraction of eve1 expression was also observed when wildtype cells were transplanted into a lateral position $(9 / 10$, not shown). However, no retraction of eve1 expression was observed when dorsal dino mutant cells were transplanted into either dorsal or lateral positions of dino mutants $(\mathrm{d} \rightarrow \mathrm{d}, 0 / 17$, Fig. $6 \mathrm{I} ; \mathrm{d} \rightarrow 1,0 / 2$, data not shown). Together these data suggest that the dino gene product acts in a non-cell-autonomous fashion and is required on the dorsal side of the early embryo to repress ventrolateral fates at early gastrula stages.

\section{Discussion}

dino and swirl regulate the dorsoventral patterning of both the mesoderm and the ectoderm

In this paper we describe several experiments carried out with two different zebrafish mutants displaying defects in early dorsoventral pattern formation, the ventralized mutant dino and the dorsalized mutant swirl. In both mutants, the dorsoventral patterning of both the mesoderm and the ectoderm is affected, and the expansion or reduction of the ventrolateral or dorsolateral mesoderm is coupled with an expansion or reduction of epidermal fates or the neuroectoderm. This indicates that dino and swirl regulate the dorsoventral patterning of both the marginal and the animal zone of the early gastrula embryo, similar to the recently demonstrated activities of the ventralizing BMPs and the dorsalizing signals from Spemann's organizer in Xenopus embryos (e.g., Sasai et al. 1995).

\section{Requirement of a BMP-like ventralizing signal and $a$ dorsal repressor of $B M P$ signaling}

Although the molecular nature of the dino and swirl genes is not known as yet, the findings provided in this paper provide compelling genetic evidence that BMP signaling is indeed required for normal ventral development in the vertebrate embryo. In swirl mutant embryos, the loss of ventral fates in derivatives of both the marginal and animal zones of the embryo is anticipated by a progressive loss of the ventrolateral expression of $B \mathrm{mp} 4$. Further, all aspects of the swirl mutant phenotype can be phenocopied and rescued by modulating BMP signaling in wild-type and mutant embryos, respectively.

Interestingly, inactivation of ventral BMP signaling in Xenopus animal caps and whole embryos by the injec- tion of mRNA encoding a dominant negative BMP receptor leads not only to a repression of epidermal and ventrolateral mesodermal cell fates, but simultaneously to an activation of neuroectodermal and dorsolateral mesodermal fates (Graff et al. 1994; Maeno et al. 1994; Suzuki et al. 1994; Sasai et al. 1995; Schmidt et al. 1995b). Consequently, the establishment of dorsal fates appears to occur by a default pathway, when ventralizing signals are absent or inhibited. During normal development, inhibition of ventralizing signals on the dorsal side of the embryo most likely is regulated by dorsalizing signals from Spemann's organizer. These include Noggin (Smith and Harland 1992), Chordin (Sasai et al. 1994), and Follistatin (Hemmati-Brivanlou et al. 1994), all of which are expressed in the organizer and which counteract BMP signaling (Sasai et al. 1995).

We provide genetic evidence indicating that Dino acts in this way in the zebrafish. Consistent with the activities of noggin and chordin in ectopic expression studies in Xenopus, Dino is required for both the dorsalization of lateral mesoderm in the marginal zone and the induction of neuroectoderm in the dorsal animal zone of the early gastrula zebrafish embryo. Both of these inductions appear to be achieved indirectly via a suppression of BMPs or related ventralizing signals. If Dino acted as an active dorsalizer, rather than as a suppressor of ventralizing signals, the dorsal deficiencies in dino mutants should not be rescued by the inactivation of ventralizing activities. However, we found that the injection of mRNA encoding a truncated BMP receptor or Noggin fully compensates for the loss of dino in mutant embryos. Even more important, the generation of dino-swirl double mutants does the same, and swirl is epistatic to dino. This suggests that dino encodes not an active dorsalizer, but rather a suppressor of a BMP or a similar swirl-dependent ventralizing activity. Moreover, as we were able to raise normal and fertile homozygous dino mutant fish after transient expression of RNAs, it would appear that dino has no essential function in later developmental processes. In the cell transplantation experiments, the transplantation of wild-type cells into the dorsal shield of early gastrula mutant embryos resulted in a retraction of ventrolateral fates and a rescue of the dino mutant phenotype. This indicates that the BMP-repressive function of Dino is required during gastrulation, the same period when BMPs have been shown to be active in $\mathrm{Xe}$ nopus embryos (Jones et al. 1996). Furthermore, Dino function appears to be restricted to the dorsal side of the embryo, the site of Spemann organizer activity. This finding makes it very unlikely that the dino mutant phenotype is caused by a gain-of-function mutation in a component of the ventralizing pathway. In sum, these data provide genetic evidence for the existence and requirement of a dorsal repressor of ventralizing signals, as proposed from embryological studies in Xenopus.

\section{The morphogenetic character of the ventralizing} activity

The antagonizing effects of dino and swirl in the double- 
mutant analysis and of dino and BMP in the injection studies appear to be dose-dependent, as suggested by the observation that the partial inactivation of ventralizing BMP signaling and the partial loss of swirl lead to a rescue of the dino mutant phenotype, while a stronger inactivation of BMP signaling and the complete loss of swirl lead to a conversion of ventralized embryos into the opposite, dorsalized phenotype. This dose-dependence points to a morphogenetic character of the signals involved in the regulation of dorsoventral pattern formation. According to our data, BMP4 or a similar swirldependent ventralizing signal functions as the dorsoventral morphogen, while the suppressive function of Dino ensures that this morphogenetic activity is indeed present in the form of a gradient along the dorsoventral axis of the early gastrula embryo, with the highest activity at the ventral and the lowest activity at the dorsal side. According to this model, positional identities along the $\mathrm{D}-\mathrm{V}$ axis are defined by the action of one, ventralizing morphogen. However, we cannot rule out the existence of an as yet unidentified opposing dorsalizing morphogen, which requires the activity of Dino to overcome the otherwise dominant activity of the ventralizing morphogen.

\section{Dino function and BMP autoregulation}

Until the molecular nature of the dino gene is determined, we can only speculate about how Dino might act in repressing BMP activity and Bmp4 expression. The non-cell-autonomous Dino function indicates that it acts as a signaling molecule or upstream of one. It might be a conventional signal that triggers the activation of a transcriptional repressor or the inactivation of a transcriptional activator of the $B m p$ gene in the dorsolateral target cells. In this case, the absence of dino in the mutant would lead automatically to the observed enhanced dorsolateral $\mathrm{Bmp} 4$ transcription. Alternatively, Dino might act similarly to Chordin and Noggin by binding BMP (Piccolo et al. 1996; Zimmerman and Harland, 1996), thereby preventing the binding of BMPs to their receptor. If this were the case, the enhanced dorsolateral Bmp4 transcription in dino mutants could be explained only if the ventralizing BMPs are positive regulators of their own expression. According to this model, the positive autoregulation would be attenuated on the dorsal side of the wild-type embryo by Dino titrating out free BMP proteins. Such a positive feedback has been suggested from ectopic expression studies in Xenopus (Jones et al. 1992) and has been described for the Bmp4 homologue decapentaplegic $(d p p)$ in the visceral mesoderm during Drosophila midgut morphogenesis (Reuter et al. 1990; Panganiban et al. 1990).

\section{Parallels in dorsoventral pattern formation among flies and vertebrates}

The mechanisms of dorsoventral pattern formation in the Drosophila embryo are well understood. Here, the secreted protein Decapentaplegic (Dpp) acts as a dorsal- izing morphogen (Padgett et al. 1987; Ferguson and Anderson 1992a) that is counteracted by the signal Short gastrulation (Sog; François et al. 1994) on the ventrolateral side of the early gastrula embryo. Dpp and Sog are structurally related to the Xenopus proteins BMP4 and Chordin, respectively, and the Drosophila and Xenopus homologues can substitute or mimic each other when ectopically expressed in the other organism (Holley et al. 1995; Schmidt et al. 1995a). These observations have led to the hypothesis that the mechanisms of dorsoventral patterning are conserved among invertebrates and vertebrates (reviewed in François and Bier 1995; Jones and Smith 1995; DeRobertis and Sasai 1996). Here, we provide genetic evidence in support of such a mechanistic conservation. Similar to dino and $B \mathrm{mp} 4$ in the zebrafish, $s o g$ is required for the refinement of $d p p$ expression in Drosophila (François et al. 1994). Furthermore, similar to dino and swirl, sog antagonizes dpp genetically in epistasis analyses (Ferguson and Anderson 1992b; Wharton et al. 1993; François et al. 1994).

\section{Materials and methods}

For all experiments, the dino allele $d_{i n}{ }^{t m 84}$ and the swirl allele $s w r^{t a 72}$ were used.

\section{In situ hybridization and immunostainings}

Whole-mount in situ hybridizations, immunostainings, and double stainings were carried out as described previously (Hammerschmidt et al. 1996b).

\section{RNA and DNA injection}

For mRNA injection, the plasmids pSP64T-tBr (Graff et al. 1994) and pSP64ßm-noggin (gift of R.M. Harland, University of California, Berkeley/ were linearized with EcoRI, and sense RNA was synthesized using the message machine kit (Ambion) according to the suppliers' instructions. Approximately $100 \mathrm{pg}$ of $\mathrm{tBr}$ mRNA or $5 \mathrm{pg}$ of nog mRNA per embryo were injected into the yolk or into single blastomeres of one- to four-cell-stage embryos as previously described (Hammerschmidt et al. 1996a).

For DNA injection, $\sim 20-40 \mathrm{pg}(2 \mathrm{nl})$ of $\mathrm{CsCl}_{2}$ gradient-purified pCSKA-BMP4 (Sasai et al. 1995) plasmid DNA was injected into the cytoplasm of one-cell-stage or both blastomeres of twocell-stage wild-type embryos.

\section{Cell transplantation}

The transplantation technique was similar to that described by Ho and Kane (1990). At the one-cell stage, donor embryos were injected with lysinated rhodamine dextran or biotinylated dextran (MW 10.000; Molecular Probes, Eugene, OR) at a concentration of $0.1 \mathrm{mg} / \mathrm{ml}$. Donor embryos were derived from crosses of two wild-type or two dino heterozygous parents. In the latter case, the donor embryos were kept after the transplantation to determine by their later phenotype if they were wild-type or dino mutant. Host embryos were derived from a $\operatorname{din} /+\times \operatorname{din} /+$ cross. Five different transplantations were carried out between donors and hosts of identical stages: (1) transplantation at the sphere stage without a priori knowledge of the position of the transplanted cells in donors and hosts; $(2-5)$ transplantation at shield stage: (2) from the dorsal shield into the dorsal shield $(\mathrm{d} \rightarrow \mathrm{d}) ;(3)$ from the dorsal shield into the ventral side $(\mathrm{d} \rightarrow \mathrm{v})$; 
(4) from the dorsal shield into a lateral position $(\mathrm{d} \rightarrow 1)$; (5) from the ventral side into the ventral side of the hosts $(v \rightarrow v)$. Chimeras that had received biotin-labeled cells were fixed at $80 \%$ epiboly and underwent standard whole-mount in situ hybridization, followed by detection of the biotin using the avidinebiotinylated peroxidase system (Vector Laboratories), as in the final step of the whole-mount immunostaining protocol (Schulte-Merker et al. 1992). Chimeric dino mutants were considered as rescued when their eve1 expression domain was clearly more ventrally restricted than in nonchimeric mutant siblings and broader than in nonchimeric wild-type siblings. Chimeras that had received rhodamine-labeled wild-type cells were analyzed at day 2 of development. Brightfield and fluorescent images were captured with a CCD cooled camera and were processed in Photoshop. Wild-type $\rightarrow$ din chimeras were judged as rescued when they were more similar to wild-type over the entire body length than their nonchimeric sibling with the mildest mutant phenotype. As a negative control of the transplantations at the shield stage to rule out that the rescue of the din mutants is caused by the injury of the shield rather than by the introduction of the wild-type cells, shield cells were taken out and implanted back into the shield of the same embryo. In no din mutant embryo treated this way $(0 / 11)$ was a weakening of the phenotype observed. No formation of a partial or complete secondary axis was observed in $\mathrm{d} \rightarrow \mathrm{v}$ transplantations, regardless of whether the recipients were $\operatorname{din}(0 / 4)$ or wild-type $(0 / 11)$. This indicates a crucial difference between transplantations to the dorsal and transplantations to the ventral side. It seems that a few wild-type cells transplanted into the dorsal shield can supplement the din mutant shield to form a normal body axis, while the induction of a secondary axis in the ectopic ventral environment requires an intact shield (Ho 1992; Shih and Fraser 1996).

\section{Acknowledgments}

We are very grateful to Drs. Christiane Nüsslein-Volhard and Mary Mullins for the permission to work on dino and swirl mutants prior to publication. Drs. Alvin Chin and Mark Fishman generously supplied us with a zebrafish $B m p 4$ cDNA fragment, and Drs. Jörg Odenthal and Christiane Nüsslein-Volhard with the $f k d 3$ cDNA prior to publication. We would like to thank Drs. Jon Graff and Douglas Melton for pSP64T-tBr, Dr. Chris Wright for pCSKA-BMP4, and Dr. Richard Harland for pCSKA-lacZ and pSP64ßm-noggin. Drs. Jean-Stéphane Joly, Stefan Schulte-Merker, and Leonard Zon provided published in situ and antibody reagents. We thank Drs. Richard Harland and Eddy De Robertis for sharing unpublished results. Work in A.P.M.'s laboratory was supported by a grant from the National Institutes of Health (NIH). M.H. was supported by Long-Term European Molecular Biology Organization and Human Frontier Science Program postdoctoral fellowships, and G.N.S. by a Life Sciences Research Foundation fellowship.

The publication costs of this article were defrayed in part by payment of page charges. This article must therefore be hereby marked "advertisement" in accordance with 18 USC section 1734 solely to indicate this fact.

\section{References}

Dale, L., G. Howes, B.M.J. Price, and J.C. Smith. 1992. Bone morphogenetic protein 4: A ventralizing factor in early $X e$ nopus development. Development 115: 573-585.

DeRobertis, E.M. and Y. Sasai. 1996. A common plan for dorsoventral patterning in Bilateria. Nature 380: 27-40.
Detrich, H.W., M.W. Kieran, F.Y. Chan, L.M. Barone, K. Yee, J.A. Rundstadler, and L.I. Zon. 1995. Intra-embryonic hematopoietic cell migration during vertebrate development. Proc. Natl. Acad. Sci. 92: 10713-10717.

Fainsod, A., H. Steinbeisser, and E.M. De Robertis. 1994. On the function of $B M P-4$ in patterning the marginal zone of the Xenopus embryo. EMBO I. 13: 5015-5025.

Ferguson, E.L. and K.V. Anderson. 1992a. decapentaplegic acts as a morphogen to organize dorsal-ventral pattern in the Drosophila embryo. Cell 71: 451-461.

- 1992b. Localized enhancement or repression of the activity of the TGF- $\beta$ family member, decapentaplegic, is necessary for dorsal-ventral pattern formation in the Drosophila embryo. Development 114: 583-597.

François, V. and E. Bier. 1995. Xenopus chordin and Drosophila short gastrulation genes encode homologous proteins functioning in dorsal-ventral axis formation. Cell 80: 19-20.

François, V., M. Solloway, J.W. O'Neill, J. Emery, and E. Bier. 1994. Dorsal-ventral patterning of the Drosophila embryo depends on a putative negative growth factor encoded by the short gastrulation gene. Genes \& Dev. 8: 2602-2616.

Graff, J.M., R.S. Thies, I.J. Song, A.J. Celeste, and D.A. Melton. 1994. Studies with a Xenopus BMP receptor suggest that ventral mesoderm-inducing signals override dorsal signals in vivo. Cell 79: 169-179.

Haffter, P., M. Granato, M. Brand, M.C. Mullins, M. Hammerschmidt, D.A. Kane, J. Odenthal, F.J.M. van Eeden, Y.-J. Jiang, C.-P. Heisenberg, R.N. Kelsh, M. Furutani-Seiki, R.M. Warga, E. Vogelsang, D. Beuchle, U. Schach, C. Fabian, and C. Nüsslein-Volhard. 1996. The identification of genes with unique and essential functions in the development of the zebrafish, Danio rerio. Development 123: 1-36.

Hammerschmidt, M., M.J. Bitgood, and A.P. McMahon. 1996a. Protein kinase $A$ is a common negative regulator of Hedgehog signaling in the vertebrate embryo. Genes \& Dev. 10: $647-658$.

Hammerschmidt, M., F. Pelegri, M.C. Mullins, D.A. Kane, F.J.M. van Eeden, M. Granato, M. Brand, M. Furutani-Seiki, P. Haffter, C.-P. Heisenberg, Y.-J. Jiang, R.N. Kelsh, J. Odenthal, R.M. Warga, and C. Nüsslein-Volhard. 1996b. dino and mercedes, two genes regulating dorsal development in the zebrafish embryo. Development 123: 95-102.

Hawley, S.H.B., K. Wünnenberg-Stapleton, C. Hashimoto, M.N. Laurent, T. Watabe, B.W. Blumberg, and K.W.Y. Cho. 1995. Disruption of BMP signals in embryonic Xenopus ectoderm leads to direct neural induction. Genes \& Dev. 9:29232935.

Hemmati-Brivanlou, A., O.G. Kelly, and D.A. Melton. 1994. Follistatin, an antagonist of activin, is expressed in the Spemann organizer and displays direct neuralizing activity. Cell 77: 283-295.

Ho, R.K. 1992. Axis formation in the embryo of the zebrafish, Brachydanio rerio. Sem. Dev. Biol. 3: 53-64.

Ho, R.K. and D.A. Kane. 1990. Cell-autonomous action of zebrafish spt-1 mutation in specific mesodermal precursors. Nature 348: 728-730.

Holley, S.A., P.D. Jackson, Y. Sasai, B. Lu, E.M. De Robertis, F.M. Hoffmann, and E.L. Ferguson. 1995. A conserved system for dorsal-ventral patterning in insects and vertebrates involving sog and chordin. Nature 376: 249-253.

Joly, J.-S., C. Joly, S. Schulte-Merker, H. Boulkebache, and H. Condamine. 1993. The ventral and posterior expression of the homeobox gene eve1 is perturbed in dorsalized and mutant embryos. Development 119: 1261-1275.

Jones, C.M. and J.C. Smith. 1995. Revolving vertebrates. Curr. Biol. 5: 574-576. 
Jones, C.M., K.M. Lyons, P.M. Lapan, W.V.E. Wright, and B.L.M. Hogan. 1992. DVR-4 (bone morphogenetic protein-4) as a posterior ventralizing factor in Xenopus mesoderm induction. Development 115: 639-647.

Jones, C.M., L. Dale, B.L.M. Hogan, C.V.E. Wright, and J.C. Smith. 1996. Bone morphogenetic protein-4 (BMP-4) acts during gastrula stages to cause ventralization of Xenopus embryos. Development 122: 1545-1554.

Maeno, M., R.C. Ong, A. Suzuki, N. Ueno, and H. Kung. 1994. A truncated bone morphogenetic protein receptor alters the fate of ventral mesoderm to dorsal mesoderm: Roles of animal pole tissue in the development of the ventral mesoderm. Proc. Natl. Acad. Sci. 91: 10260-10264.

Mishina, Y., A. Suzuki, N. Ueno, and R.R. Behringer. 1995. Bmpr encodes a type I bone morphogenetic protein receptor that is essential for gastrulation during mouse embryogenesis. Genes \& Dev. 9: 3027-3037.

Mullins, M.C., M. Hammerschmidt, D.A. Kane, M. Brand, F.J.M. van Eeden, M. Furutani-Seiki, M. Granato, P. Haffter, C.-P. Heisenberg, Y.-J. Jiang, R.N. Kelsh, J. Odenthal, R.M. Warga, and C. Nüsslein-Volhard. 1996. Genes establishing dorsal-ventral pattern formation in the zebrafish embryo: The ventral specifying genes. Development 123: 81-93.

Nakamaru, T., K. Takio, Y. Eto, H. Shibai, K. Titani, and H. Sugino. 1989. Activin-binding protein from rat ovary is Follistatin. Science 247: 836-838.

Padgett, R.W., R.D. St. Johnston, and W.M. Gelbart. 1987. A transcript from a Drosophila pattern gene predicts a protein homologous to the transforming growth factor- $\beta$ family. $\mathrm{Na}$ ture 325: 81-84.

Panganiban, G.E.F., R. Reuter, M.P. Scott, and F.M. Hoffmann. 1990. A Drosophila growth factor homolog, decapentaglegic regulates homeotic gene expression within and across germ layers during midgut morphogenesis. Development 110: 1041-1050.

Piccolo, S., Y. Sasai, B. Lu, and E.M. De Robertis. 1996. A possible molecular mechanism for Spemann organizer function: Inhibition of ventral signals by direct-binding of Chordin to BMP-4. Cell 86: 589-598.

Reuter, R., G.E.F. Panganiban, F. Hoffmann, and M.P. Scott. 1990. Homeotic genes regulate the spatial expression of putative growth factors in the visceral mesoderm of Drosophila embryos. Development 110: 1031-1040.

Sasai, Y., B. Lu, H. Steinbeisser, D. Geissert, L.K. Gont, and E.M. De Robertis. 1994. Xenopus chordin: A novel dorsalizing factor activated by organizer-specific homeobox genes. Cell 79: 779-790.

Sasai, Y., B. Lu, H. Steinbeisser, and E.M. De Robertis. 1995. Regulation of neural induction by the chd and Bmp-4 antagonistic patterning signals in Xenopus. Nature 376: 333-336.

Schmidt, J.E., V. François, E. Bier, and D. Kimelman. 1995a. Drosophila short gastrulation induces an ectopic axis in Xenopus: Evidence for conserved mechanisms of dorsal-ventral patterning. Development 121: 4319-4328.

Schmidt, J.E., A. Suzuki, N. Ueno, and D. Kimelman. 1995b. Localized BMP-4 mediates dorsal/ventral patterning in the early Xenopus embryo. Dev. Biol. 169: 37-50.

Schulte-Merker, S., R.K. Ho, B.G. Herrmann, and C. NüssleinVolhard. 1992. The protein product of the zebrafish homologue of the mouse $T$ gene is expressed in nuclei of the germ ring and the notochord of the early embryo. Development 116: 1021-1032.

Schulte-Merker, S., M. Hammerschmidt, D. Beuchle, K.W. Cho, E.M. DeRobertis, and C. Nüsslein-Volhard. 1994. Expression of the zebrafish goosecoid and no tail gene products in wildtype and mutant ntl embryo. Development 120: 843-852.
Shih, J. and S.E. Fraser. 1996. Characterization of the zebrafish organizer: Microsurgical analysis at the early-shield stage. Development 122: 1313-1322.

Sive, H.L. 1993. The frog prince-ss: A molecular formula for dorsoventral patterning in Xenopus. Genes \& Dev. 7: 1-12.

Smith, W.C. and R.M. Harland. 1992. Expression cloning of noggin, a new dorsalizing factor localized in the Spemann organizer in Xenopus embryos. Cell 70: 829-840.

Stachel, S.E., D.J. Grunwald, and P.Z. Myers. 1993. Lithium perturbation and goosecoid expression identify a dorsal pecification pathway in the pregastrula zebrafish. Development 117: 1261-1274.

Steinbeisser, H., A. Fainsod, C. Niehrs, Y. Sasai, and E.M. De Robertis. 1995. The role of gsc and BMP-4 in dorsal-ventral patterning of the marginal zone in Xenopus: A loss-of-function study using antisense RNA. EMBO /. 14: 5230-5243.

Strähle, U., P. Blader, D. Henrigue, and P.W. Ingham. 1993. Axial, a zebrafish gene expressed along the developing body axis, shows altered expression in cyclops mutant embryos. Genes \& Dev. 7: 1436-1446.

Suzuki, A., R.S. Thies, N. Yamayi, J.J. Song, J.M. Wozney, K. Murakami, and N. Ueno. 1994. A truncated bone morphogenetic protein receptor affects dorsal-ventral patterning in the early Xenopus embryo. Proc. Natl. Acad. Sci. 91: 1025510259.

Thisse, C., B. Thisse, M.E. Halpern, and J.H. Postlethwait. 1994. goosecoid expression in neuroectoderm and mesendoderm is disrupted in zebrafish cyclops gastrulas. Dev. Biol. 164: 420429.

Wharton, K.A., R.P. Ray, and W.M. Gelbert. 1993. An activity gradient of decapentaplegic is necessary for the specification of dorsal pattern elements in the Drosophila embryo. Development 117: 807-822.

Wilson, P.A. and A. Hemmati-Brivanlou. 1995. Induction of epidermis and inhibition of neural fate by Bmp-4. Nature 376: 331-333.

Winnier, G., M. Blessing, P.A. Labosky, and B.L.M. Hogan. 1995. Bone morphogenetic protein-4 is required for mesoderm formation and patterning in the mouse. Genes \& Dev. 9: 2105-2116.

Zimmerman, L.B. and R.M. Harland. 1996. Bmp-4 function is blocked by high affinity binding to the Spemann organizer signal Noggin. Cell 86: 599-606. 


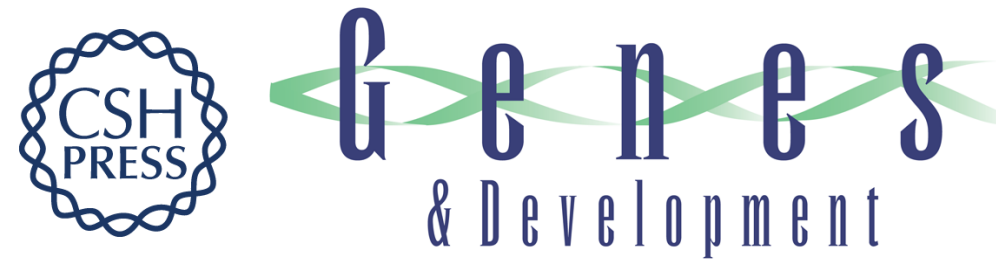

\section{Genetic analysis of dorsoventral pattern formation in the zebrafish: requirement of a BMP-like ventralizing activity and its dorsal repressor.}

M Hammerschmidt, G N Serbedzija and A P McMahon

Genes Dev. 1996, 10:

Access the most recent version at doi:10.1101/gad.10.19.2452

References This article cites 47 articles, 25 of which can be accessed free at: http://genesdev.cshlp.org/content/10/19/2452.full.html\#ref-list-1

License

Email Alerting Service

Receive free email alerts when new articles cite this article - sign up in the box at the top right corner of the article or click here.

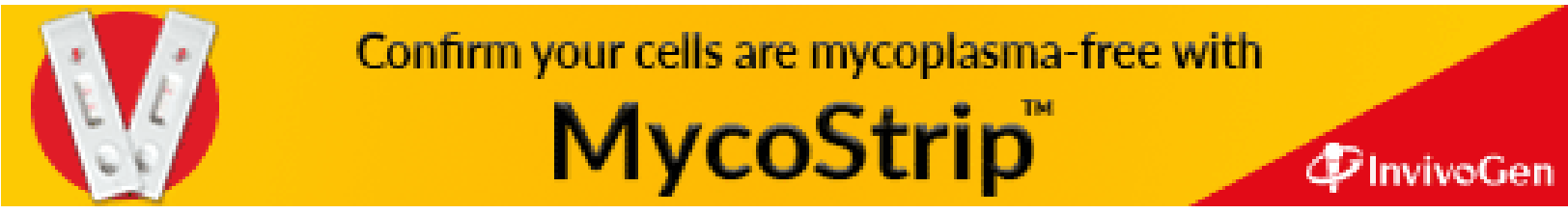

